Works of the Faculty of Forestry

University of Sarajevo

No. 1, 2015 (87-102)

UDK 595.796(497.6)

\title{
DIVERSITY AND AUTECOLOGY OF WOOD ANTS IN CENTRAL BOSNIA AND HERZEGOVINA (FORMICIDAE: FORMICA S. STR.)
}

\author{
Diverzitet i autekologija šumskih mrava u centralnoj \\ Bosni i Hercegovini (Formicidae: Formica s. str.)
}

\begin{abstract}
Adi Vesnil ${ }^{1}$, Rifat Ǵkrijelj ${ }^{1}$, Sadbera Troǵiĺ-Borovac ${ }^{1}$

In this paper new data on diversity of wood ants in Bosnia and Herzegovina are presented. First record of Formica truncorum Fabricius, 1804 from Dubogica is reported for Bosnia and Herzegovina. With this new finding total number of species from subgenus Formica s. str. known in Bosnia and Herzegovina is raised on five. Analysis of wood ants in central Bosnia indicated presence of Formica rufa Linnaeus, 1761 on mountains Trebevic, Ozren, Perun while Formica polyctena Förster, 1850 was collected only on Mt. Lemerska.

Morphometrical and ethological intermediary indicate different Formica rufa phenotypes or hybrid Formica rufa x polyctena in investigated area. Polydomy was observed in Formica polyctena and F. rufa. On the mountain Perun 30 colonies of Formica rufa in one kilometer transect were found, which represents the highest known density in Bosnia-Herzegovina.
\end{abstract}

Key words: Formica, wood ants, Bosnia, coniferous forests, conservation.

\section{INTRODUCTION - Uvod}

In Europe Formica rufa group is represented by nine species (RADCHENKO, 2015). Formica rufa Linnaeus, 1761, F. polyctena Förster, 1850, $F$. pratensis and $F$. lugubris are reported for Bosnia and Herzegovina (VESNIĹ, 2011). Extensive ecological studies of forest ants on Mount Igman were carried out during the 60's of the last century (LUTERǴEK, 1970).

Wood ants, Formica rufa species group are important ecological component of conifer forest fauna. Colonies of wood ants have up to six million workers and they are important in regulation of plant eating insects (GöSSWALD, 1981; SEIFERT, 2007). Coniferous woodlands are under strong impact by human activities. Forests of spruce and fir in Bosnia and Herzegovina are primary habitats of Formica rufa and F. polyctena. Survival of wood ants is closely linked to survival of conifer forests. One of one of the major threats to the forest is logging (THOR, 1998). Due continuous coniferous woodland deforestation and ecosystem degradation it is important to collect ecological data, information about distribution of wood ants in Bosnia and

\footnotetext{
${ }^{1}$ Faculty of Natural Sciences and Mathematics
} 
Herzegovina. Besides data on the presence of Formica rufa and F. polyctena in Bosnia and Herzegovina no additional information on biology and distribution are known. Complex taxonomy of Formica rufa and F. polyctena is based on number of hairs and cuticle sculpture and does not allow rapid identification (COLLINGWOOD AND Agosti, 1986; CZECHOWSKI AND DouWES, 1996; SOVARI, 2006).

Discrimination of Formica rufa and $F$. polyctena is difficult due character overlap (SORVARI, 2006). Intraspecific variation character overlap can be result of hybridization between Formica rufa and $F$. polyctena. In the Europe different phenotypes and even hybrids between Formica rufa and $F$. polyctena are described (Seifert, 1991; Seifert, 1992; Seifert, 2007). Although the Formica rufa and F. polyctena species were previously reported for Bosnia and Hercegovina there were no data on the possible existence of intermediary phenotypes or hybrid colonies of Formica rufa x polyctena (according to SEIFERT, 2007) or morphs described in earlier studies (SEIFER, 1991). To confirm the existence of different phenotypes in Bosnia and Herzegovina we investigated inter and intraspecific variability in Formica rufa and F. polyctena.

We also evaluated discrimination between Formica rufa and $F$. polyctena based on subjective and numerical taxonomic characters. Main anthropogenic pressures were evaluated.

\section{MATERIAL AND METHODS - Materijal i metode rada}

Investigated area obtained mountains and woodland complexes of spruce and fir in central parts of Bosnia and Herzegovina. Investigations of Dubogica (lat. 44.237 ; long. $18.313^{\circ}$ ) were conducted earlier in 2009. Formica trunctorum: 19. 06. 2009; 7 workers. In workers frontal triangle is dull; whool body covered by hairs; second and third antennal scape segments twice as long as broad (Figure 1-2.), identified according to Agosti and Collingwood, 1987.

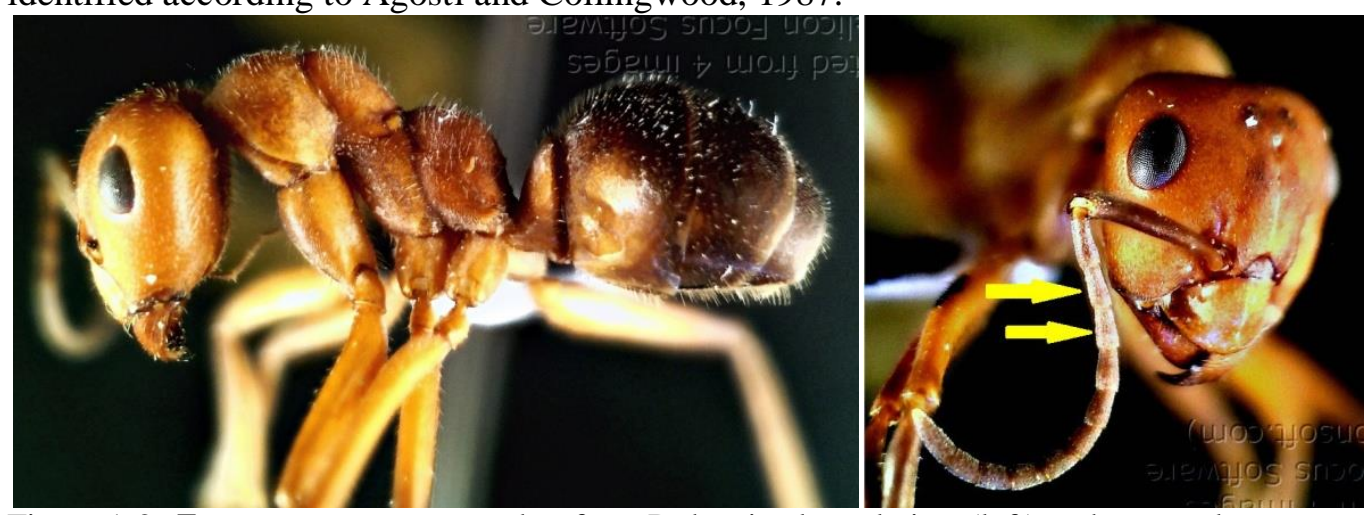

Figure 1-2: Formica trunctorum worker from Dubogica lateral view (left) and antennal scape picture show elongated third and second segment in workers (right)

Slike 1-2: Formica trunctorum radilica iz Duboštice, bočni pogled (lijevo) a slika pipaka pokazuje izduženi treći i drugi segment kod radilica (desno) 
For the Formica s. str. sample identification we used taxonomical key (AGOSTI AND ColLINGWOOd, 1987; SEIFERT, 2007).

Ecological research of Formica rufa and $F$. polyctena were conducted from 01. May till 30. October 2014. Ecological data on Formica rufa Linnaeus, 1761 and F. polyctena Förster, 1850 were obtained for the area of mountains Trebevil (lat.

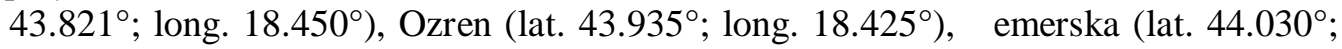
long. $18.317^{\circ}$ ) and Perun (lat. $44.160^{\circ}$; long. $18.298^{\circ}$ ).

During field investigations active methods of collecting were used. In order to analyze structural ecological data the field protocol was applied (AGOSTI ET AL 2000). Data in the field protocol included: locality, sample cod, GPS coordinate, altitude, woodland type (coniferous, deciduous, mixed forest), number of trees around discovered anthill. Number of trees was counted in two perpendicular transects $100 \mathrm{~m}$ in length with anthill in center, placement of ant colony in (deep wood, wood edge, meadow, clearings in wood made by construction of wood roads), pressures on habitat (fire, road building infrastructure, wood exploitation, expansion of settlements). Dimensions of anthills for Formica rufa and F. polyctena species were measured: length of longest anthill side (K1), length of shortest anthill side (K2), maximal (R1) and minimal (R2) diameter of colony base. Exposition of an anthill was analyzed.

The protocol was applied on each newly found colony. In laboratory workers were dry mounted on cardboard triangles and reproductive specimens were pinned on entomological needle. Morphometry was performed on Reichert stereo zoom microscope, with micrometer mounted on right ocular. Calibration of ocular was conducted on Reichert. Calibration of microoculare on slide 1 notch $=10 \mu \mathrm{m}$. Division of scale in ocular at $100 \mathrm{X}$ magnification was $13,66248 \mu \mathrm{m}$ and on $50 \mathrm{X}$ magnification 23,529412 $\mu \mathrm{m}$.

From 01. May till 30. October 2014. we found and sampled 122 nests of Formica rufa and three colonies of Formica polyctena. Workers and reproductive caste were sampled and stored in $96 \%$ ethyl alcohol. Five to ten workers were collected form each colony. We analyzed 97 workers of Formica rufa from 32 colonies and 20 workers of Formica polyctena from three colonies.

Morphometric analysis included: head with (HW), head length (HL), frontal carina with (HCL), scape length (SL), maximal scape width (SMAX), and length of longest hair on gula (CUHL), length of longest hair on pronotum (PNHL). Unilateral number of hairs on occiput $(\mathrm{nCH})$, gula $(\mathrm{nCU})$ and pronotum $(\mathrm{nPN})$ were counted. Morphometricall indices analyzed in this paper were computed: $\mathrm{CI}=\mathrm{HW} / \mathrm{HL}, \mathrm{SCI}=$ SL/SMAX.

Analyzed morphometric characteristics and indices are defined by Seifert(2007). Presence and distribution of hairs on mesopleuron was analyzed. We also analyzed micro sculpture and glossiness of frons in worker caste (COLLINGWOOD, 1979; Agosti AND Collingwood, 1987). Determination of the collected wood antôs 
material was based on subjective characteristics (AGOSTI AND COLLINGWOOD, 1987; GosswALD, 1981) and morphological indices (Table 1) (SEIFERT, 2007).

All findings of colonies identified as hybrid Formica rufa $x$ polyctena or phenotypes of $F$. rufa. In ecological analysis specimens were treated as Formica rufa .

Statistical analysis included descriptive statistic and inferential ANOVA, post hoc Newman-Keules test. Differences in frequencies were tested by Kruskal Wallis test. Multivariate analysis included discriminant function. Gradient analysis was performed by PCA analysis. Population density was calculated as transect length defined by minimal and maximal altitude of findings divided by number of colonies.

Table 1:Morphometric indices for identification of wood ants species and hybrid according to (SEIFERT, 2007)

Tabela 1: Morfometrijski pokazatelji korišteni u identifikaciji vrsta i hibrida šumskih mrava prema (SEIFERT, 2007)

\begin{tabular}{|r|r|r|}
\hline Formica rufa & Formica rufa $\boldsymbol{x}$ polyctena & Formica polyctena \\
\hline $\mathrm{nCU}=5.10-11.00$ & $\mathrm{nCU}=0.19-6.60$ & $\mathrm{nCU}=0.10-3.00$ \\
\hline $\mathrm{CUHL}=155.00-224.00$ & $\mathrm{CUHL}=96.00-197.00$ & $\mathrm{CUHL}=7.00-79.00$ \\
\hline $\mathrm{nPN}=12.50-45.00$ & $\mathrm{nPN}=5.80-16.00$ & $\mathrm{nPN}=0.1-5.6$ \\
\hline $\mathrm{PNHL}=61.00-102.00$ & $\mathrm{PNHL}=47.0-83.00$ & $\mathrm{PNHL}=6.00-55.00$ \\
\hline $\mathrm{nCH}=0.00-3.60$ & $\mathrm{nCH}=0.00-1.20$ & $\mathrm{nCH}=0.00-0.90$ \\
\hline $\mathrm{SI}=\mathrm{SL} / \mathrm{SMAX}=10.13 \pm 0.29$ & $\mathrm{SI}=\mathrm{SL} / \mathrm{SMAX}=10.00 \pm 0.24$ & $\mathrm{SI}=\mathrm{SL} / \mathrm{SMAX}=10.00 \pm 0.24$ \\
\hline
\end{tabular}

\section{RESULTS AND DISCUSSION - Rezultati rada i diskusija}

First finding of Formica truncorum Fabricius, 1804 was obtained earlier in 2009. from faunistic research of Duboġica (municipality Vareg். Field investigations in 2014. confirmed presence of Formica rufa Linnaeus, 1761, F. polyctena Förster, 1850 and $F$. pratensis in Bosnia and Herzegovina. With additional new finding of Formica truncorum, a total number of species belonging to Formica rufa group in Bosnia and Herzegovina is raised up to four. In Serbia number of Formica rufa group species is same as in Bonia (Petrov, 2006). In Croatia and Montenegro Formica rufa group is represented by five species (BRAL, KO, 2006; KARAMAN, 2004).

Analysis based on seta number indicated presence of two different phenotypes of Formica rufa in researched area. Typical Formica rufa phenotypes were found in colonies with the head width of worker caste higher than $1.8 \mathrm{~mm}$ (Table 7). Typical Formica rufa has the highest average unilateral seta number on occiput, gula and pronotum (Table 7).

Second Formica rufa phenotype is with significantly $(\mathrm{p}<5 \%)$ narrower head with $1,7 \mathrm{~mm}$ (Tab. 7.). Compared to Formica rufa with typical pilosity second F. rufa phenotype has fewer hairs on pronotum and gula (Table 7). The fact that ants with smaller head were with less number of hairs is important. There is significant size dependent decline of pilosity in very small workers (SEIFERT, 1991). Juvenile colonies 
with smaller worker caste exhibit decline of pilosity and were included in analysis. In analysis only five workers were with head width smaller than $1.4 \mathrm{~mm}$. Correlation between head width and number of seta was positive, but linear regression was not statistically significant at $5 \%$ level, $\mathrm{nPN}=-16.60+0.01806 * \mathrm{HW}$, correlation $\mathrm{r}=$ 0.50492 .

Table 2: Frequency table of seta number on occiput (nCH) for Formica rufa and F. rufa phenotype

Tabela 2: Distribucija frekvencija za broj dlaka na podbratku (nCH) za Formica rufa i F. rufa fenotip

\begin{tabular}{|l|c|c|l|c|c|}
\hline \multicolumn{3}{|c|}{ Formica rufa } & \multicolumn{3}{c|}{ Formica rufa phenotype } \\
\multicolumn{1}{|c|}{ K-S d=.53703, $\mathbf{p}<. \mathbf{0 1}$; Lilliefors $\mathbf{p}<. \mathbf{0 1}$} & \multicolumn{1}{|c|}{ K-S d=.53336, $\mathbf{p}<. \mathbf{0 1}$; Lilliefors $\mathbf{p}<. \mathbf{0 1}$} \\
\hline Kategory & Count & Percent & Kategory & Count & Percent \\
\hline$-0.5<\mathrm{x}<=\mathbf{0 . 0}$ & 44 & 97.7 & $-0.5<\mathrm{x}<=\mathbf{0 . 0}$ & 49 & 94.2 \\
\hline $0.5<\mathrm{x}<=\mathbf{1 . 0}$ & 0 & 0.0 & $0.5<\mathrm{x}<=\mathbf{1 . 0}$ & 2 & 3.8 \\
\hline $1.5<\mathrm{x}<=\mathbf{2 . 0}$ & 1 & 2.3 & $1.5<\mathrm{x}<=\mathbf{2 . 0}$ & 1 & 1.9 \\
\hline Missing & 0 & 0.0 & Missing & 0 & 0.0 \\
\hline
\end{tabular}

Number of seta on gula show statistically significant difference between Formica polyctena and two Formica rufa phenotypes (Table 3-4).

Table 3: Multiple Comparisons p values (2-tailed) for number of seta on gula (nCU) KruskalWallis test: $\mathrm{H}(2, \mathrm{~N}=117)=11.07274 \mathrm{p}=.0039$, statistically significant differences are written in bold

Tabela 3: Višestruko dvosmjerno poređenje p vrijednosti za broj seta na podbratku (nCU) Kruskal-Wallis test: $H(2, N=117)=11,07274 p=, 0039$, statistički značajne razlike su podebljane

\begin{tabular}{|l|c|c|c|}
\hline \multirow{2}{*}{ Species } & \multicolumn{3}{|c|}{ Species } \\
\cline { 2 - 4 } & Formica rufa & F. rufa phenotype & F. polyctena \\
\hline Formica rufa & & 0.291821 & $\mathbf{0 . 0 0 6 3 2 2}$ \\
\hline Formica rufa phenotype & 0.291821 & & $\mathbf{0 . 0 3 5 2 9 8}$ \\
\hline Formica polyctena & $\mathbf{0 . 0 0 6 3 2 2}$ & $\mathbf{0 . 0 3 5 2 9 8}$ & \\
\hline
\end{tabular}


Table 4: Frequency table for number of seta on gula (nCU) for Formica rufa and F. rufa phenotype

Tabela 4: Distribucija frekvencija za broj dlaka na podbratku (nCU) za Formica rufa i F. rufa fenotip

\begin{tabular}{|l|c|c|l|c|c|}
\hline \multicolumn{3}{|c|}{ Formica rufa } & \multicolumn{3}{c|}{ Formica rufa phenotype } \\
\multicolumn{1}{|c|}{ K-S d=.15259, $\mathbf{p}>\mathbf{. 2 0}$; Lilliefors $\mathbf{p}<\mathbf{. 0 5}$} & \multicolumn{2}{c|}{ K-S d=.18410, $\mathbf{p}<\mathbf{1 0}$ Lilliefors $\mathbf{p}<. \mathbf{0 1}$} \\
\hline Kategory & Count & Percent & Kategory & Count & Percent \\
\hline $0.0<\mathrm{x}<=\mathbf{1 . 0}$ & 3 & 6.66667 & $-1.0<\mathrm{x}<=\mathbf{0 . 0}$ & 4 & 7.69231 \\
\hline $1.0<\mathrm{x}<=\mathbf{2 . 0}$ & 6 & 13.33333 & $0.0<\mathrm{x}<=\mathbf{1 . 0}$ & 4 & 7.69231 \\
\hline $2.0<\mathrm{x}<=\mathbf{3 . 0}$ & 9 & 20.00000 & $1.0<\mathrm{x}<=\mathbf{2 . 0}$ & 5 & 9.61538 \\
\hline $3.0<\mathrm{x}<=\mathbf{4 . 0}$ & 10 & 22.22222 & $2.0<\mathrm{x}<=\mathbf{3 . 0}$ & 16 & 30.76923 \\
\hline $4.0<\mathrm{x}<=\mathbf{5 . 0}$ & 11 & 24.44444 & $3.0<\mathrm{x}<=\mathbf{4 . 0}$ & 12 & 23.07692 \\
\hline $5.0<\mathrm{x}<=\mathbf{6 . 0}$ & 5 & 11.11111 & $4.0<\mathrm{x}<=\mathbf{5 . 0}$ & 6 & 11.53846 \\
\hline $6.0<\mathrm{x}<=7.0$ & 1 & 2.22222 & $5.0<\mathrm{x}<=6.0$ & 5 & 9.61538 \\
\hline Missing & 0 & 0.00000 & Missing & 0 & 0.00000 \\
\hline
\end{tabular}

Less than 5\% of workers of Formica rufa were with hairs on occiput and all F. polyctena workers were without hairs. Multiple comparisons Kruskal-Wallis test: $\mathrm{H}$ $(2, \mathrm{~N}=117)=.8735411 \mathrm{p}=.6461$ did not show statistically significant differences in frequencies for number of seta on occiput $(\mathrm{nCH})$ between Formica rufa, F. rufa phenotype and Formica polyctena. (Table 2).

Highest number of seta on pronotum was in workers of Formica rufa. Differences in seta number are statistically significant between Formica rufa, F. rufa phenotype and $F$. polyctena (Table 5-6).

Table 5: Multiple Comparisons p values (2-tailed); number of seta on pronotum (nPN) Kruskal-Wallis test: $\mathrm{H}(2, \mathrm{~N}=117)=20.58424 \mathrm{p}=.0000$, statistically significant differences are written in bold

Tabela 5: Višestruko dvosmjerno poređenje p vrijednosti za broj seta na pronotumu (nCU) Kruskal-Wallis test: $H(2, N=117)=20,58424 p=, 0000$, statistički značajne razlike su podebljane

\begin{tabular}{|l|c|c|c|}
\hline \multirow{2}{*}{ Species } & \multicolumn{3}{|c|}{ Species } \\
\cline { 2 - 4 } & Formica rufa & F. rufa phenotype & F. polyctena \\
\hline Formica rufa & & $\mathbf{0 . 0 0 1 6 8 6}$ & $\mathbf{0 . 0 0 1 2 8 6}$ \\
\hline Formica rufa phenotype & $\mathbf{0 . 0 0 1 6 8 6}$ & & 0.055738 \\
\hline Formica polyctena & $\mathbf{0 . 0 0 1 2 8 6}$ & 0.055738 & \\
\hline
\end{tabular}


Table 6: Frequency table for number of seta on pronotum (nPN) for Formica rufa and F. rufa phenotype

Tabela 6: Distribucija frekvencija za broj dlaka na pronotumu (nPN) za Formica rufa i F. rufa fenotip

\begin{tabular}{|l|c|c|l|c|c|}
\hline \multicolumn{3}{|c|}{ Formica rufa } & \multicolumn{3}{c|}{ Formica rufa phenotype } \\
\multicolumn{2}{|c|}{ K-S d=.09456, $\mathbf{p}>\mathbf{. 2 0}$; Lilliefors $\mathbf{p}>\mathbf{. 2 0}$} & \multicolumn{2}{|c|}{ K-S d=.13012, $\mathbf{p}>\mathbf{. 2 0}$; Lilliefors $\mathbf{p}<. \mathbf{0 5}$} \\
\hline Kategory & Count & Percent & Kategory & Count & Percent \\
\hline$-5.0<\mathrm{x}<=\mathbf{0 . 0}$ & 0 & 0.00000 & $-5.0<\mathrm{x}<=\mathbf{0 . 0}$ & 0 & 0.00000 \\
\hline $0.0<\mathrm{x}<=\mathbf{5 . 0}$ & 3 & 6.66667 & $0.0<\mathrm{x}<=\mathbf{5 . 0}$ & 10 & 19.23077 \\
\hline $5.0<\mathrm{x}<=\mathbf{1 0 . 0}$ & 5 & 11.11111 & $5.0<\mathrm{x}<=\mathbf{1 0 . 0}$ & 10 & 19.23077 \\
\hline $10.0<\mathrm{x}<=\mathbf{1 5 . 0}$ & 7 & 15.55556 & $10.0<\mathrm{x}<=\mathbf{1 5 . 0}$ & 17 & 32.69231 \\
\hline $15.0<\mathrm{x}<=\mathbf{2 0 . 0}$ & 11 & 24.44444 & $15.0<\mathrm{x}<=\mathbf{2 0 . 0}$ & 7 & 13.46154 \\
\hline $20.0<\mathrm{x}<=\mathbf{2 5 . 0}$ & 11 & 24.44444 & $20.0<\mathrm{x}<=\mathbf{2 5 . 0}$ & 5 & 9.61538 \\
\hline $25.0<\mathrm{x}<=\mathbf{3 0 . 0}$ & 7 & 15.55556 & $25.0<\mathrm{x}<=\mathbf{3 0 . 0}$ & 2 & 3.84615 \\
\hline $30.0<\mathrm{x}<=\mathbf{3 5 . 0}$ & 1 & 2.22222 & $30.0<\mathrm{x}<=\mathbf{3 5 . 0}$ & 1 & 1.92308 \\
\hline Missing & 0 & 0.00000 & Missing & 0 & 0.00000 \\
\hline
\end{tabular}

Morphological analysis of workers showed that $54.6 \%$ of analyzed colonies were with intermediary characteristics of Formica rufa and $F$. polyctena. In literature maximal established percentage of hybrids was $26.0 \%$ (SEIFERT, 2007). Morphometric analysis based on description of hybrid Formica rufa x polyctena SEIFERT (2007) did not indicate statistically significant difference in length of seta on gula and pronotum between Formica rufa and assumed hybrid colonies (Graphs 1-2).
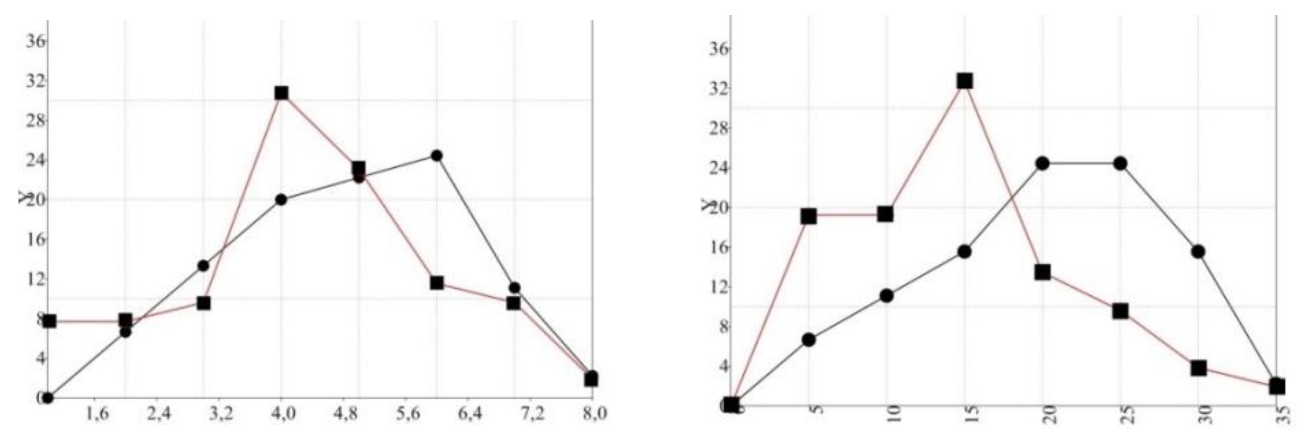

Graph 1-2: Distribution of seta number plotted on y axis; gula (left) and pronotum (right) in Formica rufa (dot) and F. rufa phenotype (square)

Grafikon 1-2: Distribucija broja seta na y osi; podbradak (lijevo) i pronotum (desno) kod Formica rufa (tačka) and F. rufa fenotip (kvadrat)

Two colonies from Perun and one colony from Lavljak show clear characteristics of hybrid Formica rufa x polyctena (SEIFERT, 2007). Determination of hybrid was based on length of seta on gula, pronotum and number of seta on pronotum. Typical characteristics of Formica rufa were found in $42.4 \%$ of colonies. Typical Formica polyctena characteristics were found in three colonies. 
Number and distribution of seta showed intraspecific and intracolonial variability. Number of worker caste with intermediary seta characteristics between Formica rufa and $F$. polyctena was $44.5 \%$. Most significant difference between Formica rufa and $F$. polyctena was in seta number at gula and pronotum. Average number of seta in colonies of Formica rufa was between 0.0 and maximal 0.7. In colonies of Formica rufa with intermediary phenotypes maximal average number of seta was 0.5 (Table 7).

Microsculpture and glossiness of frons in worker caste proposed by COLlingwoOd (1979) and AGOSTI AND COLLINGWOOd (1987) was impractical and for Bosnian wood ants characters were poorly defined. Intensity of shines of the frons in samples shows intracolonial variation. Similar data were obtained for seta number and distribution of seta on mesopleuron. Number of seta on mesopleuron also showed intracolonial variability. In same colonies workers without hairs on mesopleuron, workers with hairs on posterior side of mesopleuron and with evenly distributed hairs were found. In worker caste seta on mesopleuron were not detected and it was stabile character in colonies of Formica polyctena.

Statistically significant differences between Formica rufa and hybrids of Formica rufa $x$ polyctena colonies were detected (Table 7). Length of scape has statistically significant difference between Formica rufa and F. polyctena (Table 7). Same result was confirmed by scape index (Table 7). 
Table 7: Morphometric indices for Formica rufa, Formica rufa phenotype and Formica polyctena: average \pm standard deviation $(\mathrm{SD})$, minimal (min), maximal (max) value, coefficient of variability (V\%), standard error mean (SE). Differences were tested by ANOVA and post-hoc Newman-Keuls test, mean values that share same letter are statistically different at $5 \%$, values that share same letter and (-) are not statistically different

Tabela 7: Pregled variranja odabranih morfometrisjkih karaktera za Formica rufa, Formica rufa fenotip i Formica polyctena: aritmetickka sredina standardna devijacija (SD), minimalna (min), maksimalna (max) vrijednost, koeficijent varijabilnosti (V\%), standardna greška aritmeticke sredine (SE). Razlike su testirane ANOVA i post-hoc Newman-Keuls testom, aritmetičke sredine koje dijele isto slovo su statistički značajne na nivou od 5\%, vrijednosti koje imaju (-) nisu značajne

\begin{tabular}{|c|c|c|c|c|c|c|c|c|c|}
\hline & \multicolumn{3}{|c|}{$\begin{array}{c}\text { Formica rufa } \\
\quad \mathrm{N}=45\end{array}$} & \multicolumn{3}{|c|}{$\begin{array}{c}\text { Formica rufa phenotype } \\
\qquad \mathrm{N}=\mathbf{5 2}\end{array}$} & \multicolumn{3}{|c|}{$\begin{array}{c}\text { Formica polyctena } \\
\mathbf{N}=\mathbf{2 0}\end{array}$} \\
\hline & $\begin{array}{l}\text { average } \pm \text { SD } \\
(\min . \max )\end{array}$ & $\mathrm{V} \%$ & $\mathrm{SE}$ & $\begin{array}{c}\text { average } \pm \text { SD } \\
(\min . \max )\end{array}$ & $\mathrm{V} \%$ & SE & $\begin{array}{c}\text { average } \pm \text { SD } \\
(\min . \max )\end{array}$ & $\mathrm{V} \%$ & SE \\
\hline $\mathrm{HW}$ & $\begin{array}{c}1821.6^{\mathrm{a}} \pm 203.4 \\
(1365.0,2235.0)\end{array}$ & 11.2 & 30.3 & $\begin{array}{c}1695.0^{\mathrm{a}-} \pm 207.0 \\
(1224.0,2071.0)\end{array}$ & 12.2 & 28.7 & $\begin{array}{c}1637.7^{\mathrm{a}-} \pm 91.7 \\
(1505.9,1788.2)\end{array}$ & 5.6 & 20.5 \\
\hline HL & $\begin{array}{c}1965.0^{\mathrm{a}} \pm 184.4 \\
(1576.0,2329.0)\end{array}$ & 9.4 & 27.5 & $\begin{array}{c}1861.8^{\mathrm{a}-} \pm 192.9 \\
(1435.0,2141.0)\end{array}$ & 10.4 & 26.8 & $\begin{array}{c}1794.1^{\mathrm{a}-} \pm 93.1 \\
(1623.5,1952.9)\end{array}$ & 5.2 & 20.8 \\
\hline $\mathrm{HCL}$ & $\begin{array}{c}602.2^{\mathrm{a}} \pm 59.2 \\
(478.0,724.0)\end{array}$ & 9.8 & 8.8 & $\begin{array}{c}556.0^{\mathrm{a}-} \pm 57.3 \\
(424.0,642.0)\end{array}$ & 10.3 & 7.9 & $\begin{array}{c}539.7^{\mathrm{a}-} \pm 51.8 \\
(464.5,628.5)\end{array}$ & 9.6 & 11.6 \\
\hline SL & $\begin{array}{c}1763.1^{\mathrm{a}} \pm 166.0 \\
(1341.0,1953.0)\end{array}$ & 9.4 & 24.7 & $\begin{array}{c}1664.4^{\mathrm{a}} \pm 164.7 \\
(1247.0,1906.0)\end{array}$ & 9.9 & 22.8 & $\begin{array}{c}1066.0^{\mathrm{a}} \pm 271.1 \\
(874.4,1788.0)\end{array}$ & 25.4 & 60.6 \\
\hline SMAX & $\begin{array}{c}216.0^{\mathrm{a}} \pm 18.0 \\
(178.0,246.0)\end{array}$ & 8.3 & 2.7 & $\begin{array}{c}202.7^{\mathrm{a}} \pm 18.9 \\
(164.0,232.0)\end{array}$ & 9.3 & 2.6 & $\begin{array}{c}207.7 \pm 16.9 \\
(177.6,232.3)\end{array}$ & 8.1 & 3.8 \\
\hline $\mathrm{nCH}$ & $\begin{array}{l}0.04 \pm 0.3 \\
(0.0,2.0)\end{array}$ & 670.8 & 0.0 & $\begin{array}{c}0.1 \pm 0.3 \\
(0.0,2.0)\end{array}$ & 434.3 & 0.05 & 0.0 & 0.0 & 0.0 \\
\hline $\mathrm{nCU}$ & $\begin{array}{c}3.9 \pm 1.5 \\
(1.0,7.0)\end{array}$ & 38.8 & 0.2 & $\begin{array}{c}3.3 \pm 1.6 \\
(0.0,6.0)\end{array}$ & 49.6 & 0.2 & 0.0 & 0.0 & 0.0 \\
\hline CUHL & $\begin{array}{c}154.9 \pm 27.3 \\
(96.0,219.0) \\
\end{array}$ & 17.7 & 4.1 & $\begin{array}{l}137.3 \pm 49.1 \\
(0.0,246.0) \\
\end{array}$ & 35.7 & 6.8 & 0.0 & 0.0 & 0.0 \\
\hline $\mathrm{nPN}$ & $\begin{array}{c}17.9 \pm 7.4 \\
(2.0,32.0) \\
\end{array}$ & 41.5 & 1.1 & $\begin{array}{c}12.6 \pm 7.0 \\
(2.0,33.0) \\
\end{array}$ & 55.5 & 1.0 & 0.0 & 0.0 & 0.0 \\
\hline PNHL & $\begin{array}{c}99.6 \pm 16.6 \\
(68.0,164.0) \\
\end{array}$ & 16.6 & 2.5 & $\begin{array}{c}89.9 \pm 16.1 \\
(55.0,123.0) \\
\end{array}$ & 17.9 & 2.2 & 0.0 & 0.0 & 0.0 \\
\hline HW/HL & $\begin{array}{c}0.93 \pm 0.035 \\
(0.9,1.0) \\
\end{array}$ & 3.72 & 0.005 & $\begin{array}{c}0.91 \pm 0.03 \\
(0.85,1.02) \\
\end{array}$ & 3.67 & 0.00 & $\begin{array}{c}0.91 \pm 0.02 \\
(0.89,0.95) \\
\end{array}$ & 1.98 & 0.00 \\
\hline SL/SMAX & $\begin{array}{l}8.17^{\mathrm{a}-} \pm 0.45 \\
(7.40,9.52) \\
\end{array}$ & 5.55 & 0.067 & $\begin{array}{l}8.22^{\mathrm{a}-} \pm 0.42 \\
(7.15,9.36) \\
\end{array}$ & 5.09 & 0.06 & $\begin{array}{c}5.16^{\mathrm{a}} \pm 1.4 \\
(4.38,8.59) \\
\end{array}$ & 27.05 & 0.31 \\
\hline
\end{tabular}

*head with (HW), head length (HL), frontal carina with (HCL), scape length (SL), maximal scape with (SMAX), maximal length of seta on gula (CUHL), maximal length of seta on pronotum (PNHL), unilateral number of hairs on occiput $(\mathrm{nCH})$, unilateral number of hairs on gula (nCU) and unilateral number of hairs on pronotum $(\mathrm{nPN})$.

Six variables were included in multivariate discriminant function analysis model. Partial Wilks' Lambda indicates that variable scape length had greatest discriminant power between Formica rufa, F. rufa phenotype and F. polyctena (Table 8). Frontal carina with and scape index did not show statistically significant discriminant power (Table 9). 
The first discriminant function is weighted most heavily by the scapus length (SL) and head width (HW). The other two variables also contribute to this function. The second function is marked by head width (HW) and head length (HL) (Table 10; Graph 3-4). The less hairy morph of Formica rufa in our investigations is by hair number very close to $F$. polyctena. Other authors reported Formica polyctena hairy phenotype from continental Europe suggesting that is hybrid of $F$. polyctena and $F$. rufa (SEIFERT, 1991; SEIFERT, 2007; CZECHOWSKI AND DouWES, 1996). SORVARI (2006) reported Formica polyctena phenotype with more hairs form Finland.

Table 8: Discriminant Function Analysis Summary, number of variables in the model: 6; groups 33; Wilks' Lambda: ,01179 approx. $\mathrm{F}(192.475)=2.7719 \mathrm{p}<0.0000$

Tabela 8: Analiza diskriminantne funkcije, broj varijabli u modelu 6; 33 grupe; Wilks' Lambda: ,01179 approx. $F(192,475)=2,7719 p<0,0000$

\begin{tabular}{|l|c|c|c|c|c|c|}
\hline & Wilks' & Partial & F-remove & p-value & Toler. & 1-Toler. \\
\hline SL & 0.019588 & 0.602138 & 1.631224 & $\mathbf{0 . 0 4 1 2 6 7}$ & 0.092671 & 0.907329 \\
\hline HW & 0.024342 & 0.484542 & 2.626266 & $\mathbf{0 . 0 0 0 2 7 3}$ & 0.001249 & 0.998751 \\
\hline HCL & 0.018109 & 0.651316 & 1.321650 & 0.159664 & 0.250144 & 0.749856 \\
\hline SL/SMAX & 0.017741 & 0.664834 & 1.244582 & 0.215487 & 0.150884 & 0.849116 \\
\hline HL & 0.024376 & 0.483865 & 2.633395 & $\mathbf{0 . 0 0 0 2 6 3}$ & 0.001652 & 0.998348 \\
\hline HW/HL & 0.023896 & 0.493591 & 2.532858 & $\mathbf{0 . 0 0 0 4 4 3}$ & 0.009580 & 0.990420 \\
\hline
\end{tabular}
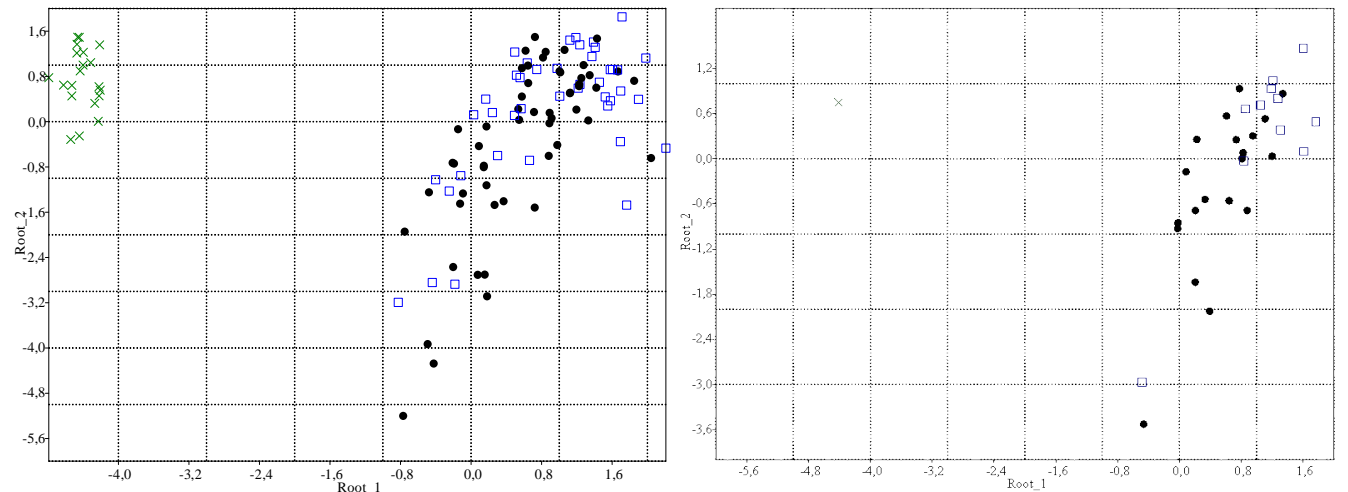

Graph 3-4: Unstandardized Canonical Scores for workers (left) and Means of Canonical Variables for colonies (right): Formica rufa (square), F. rufa phenotype (dot) and $F$. polyctena $(\mathrm{x})$

Grafikon 3-4: Nestandardizirane kanonijske vrijednosti za radnike (lijevo) i srednje vrijednosti kanonijskih varijabli za kolonije (desno): Formica rufa (kvadrat), F. rufa fenotip (tačka) i F. polyctena (x) 
Table 9: Chi square test of successive roots, statistically significant roots are in bold Tabela 9: Hi-kvadrat test sukcesivnih korijena, statistički značajni korijeni su podebljani

\begin{tabular}{|l|c|c|c|c|c|c|}
\hline Roots Removed & Eigen- & Canonicl & Wilks' $^{\prime}$ & Chi-Sqr. & df & p-value \\
\hline 0 & 4.119746 & 0.897038 & 0.011795 & 428.4682 & 192 & $\mathbf{0 . 0 0 0 0 0 0}$ \\
\hline 1 & 1.522614 & 0.776908 & 0.060387 & 270.8736 & 155 & $\mathbf{0 . 0 0 0 0 0 0}$ \\
\hline 2 & 1.058620 & 0.717104 & 0.152333 & 181.5826 & 120 & $\mathbf{0 . 0 0 0 2 4 4}$ \\
\hline 3 & 0.652521 & 0.628382 & 0.313596 & 111.9061 & 87 & 0.037328 \\
\hline 4 & 0.478115 & 0.568738 & 0.518224 & 63.4340 & 56 & 0.230882 \\
\hline 5 & 0.305491 & 0.483741 & 0.765995 & 25.7249 & 27 & 0.533904 \\
\hline
\end{tabular}

Table 10: Standardized Coefficients for Canonical Variables Tabela 10: Standardizirani koeficijenti kanonijskih varijabli

\begin{tabular}{|l|c|c|c|c|c|c|}
\hline & Root 1 & Root 2 & Root 3 & Root 4 & Root 5 & Root 6 \\
\hline SL & 1.24509 & 0.6208 & -0.6844 & -0.09393 & -2.60778 & 1.25598 \\
\hline HW & 2.11301 & -14.2479 & 22.8875 & -0.92105 & -3.10736 & 7.67676 \\
\hline HCL & -0.22987 & -0.1541 & 0.4068 & -1.50475 & -0.31567 & -1.17970 \\
\hline SL/SMAX & -0.02773 & -0.7747 & 0.3257 & 0.17654 & 2.14995 & -1.12572 \\
\hline HL & -2.07499 & 12.7865 & -19.4089 & 1.60565 & 4.68553 & -6.03722 \\
\hline HW/HL & -0.75005 & 5.3270 & -7.8290 & 1.34339 & 1.33152 & -3.25133 \\
\hline Eigenval & 4.11975 & 1.5226 & 1.0586 & 0.65252 & 0.47811 & 0.30549 \\
\hline Cum.Prop & 0.50629 & 0.6934 & 0.8235 & 0.90370 & 0.96246 & 1.00000 \\
\hline
\end{tabular}

Ecological data between Formica rufa and F. polyctena were not comparable due small number of $F$. polyctena findings. Statistically significant difference in altitude between Formica polyctena and F.rufa, was detected by multiple comparisons $\mathrm{p}$ values Kruskal-Wallis test at 5\% significance (Table 11). Differences in anthill dimensions between species were not detected at 5\% significance level by NewmanKeuls test. Findings of Formica polyctena were on lower boundary of Formica rufa vertical distribution.

Formica rufa and $F$. polyctena were most common in coniferous forests. Relative number of colonies of Formica rufa and its phenotype in coniferous Abietopicetum forest habitat was between $88 \%$ and $89 \%$. All colonies of Formica polyctena were in Abieto-picetum woodlands. Formica rufa builds colonies more often near wood roads. Total number of Formica rufa colonies near road was $91 \%$, in regard to Formica rufa phenotype $61 \%$. Colonies of Formica polyctena were not found near roads. Data from LUTERǴEK (1960) indicate that Formica polyctena is infrequent, compared to Formica rufa. Distribution data from Igman indicate that large and small wood ant are more common in meadows than in the woods (LUTERGEKK, 1960).

Colonies of Formica polyctena were found in deep woodland habitats $34 \%$ and $66 \%$ in congregation with more than 30 trees. Colonies of Formica rufa also show strong association with dense forests. Most colonies 86\% of Formica rufa were collected in habitats with 20 to 30 trees, similar number of colonies $72 \%$ were for Formica rufa phenotype (Graph 5-6). 
Table 11: Altitude and anthill dimension for Formica rufa and F. polyctena colonies average \pm standard deviation $(\mathrm{SD})$, minimal $(\mathrm{min})$, maximal (max) value, coefficient of variability (V\%), standard error mean (SE) Differences were tested by ANOVA and post-hoc Newman-Keuls test, mean values that share same letter are statistically different at 5\%, values that share same letter and (-) are not statistically different

Tabela 11: Nadmorska visina i dimenzije kolonija za Formica rufa i Formica polyctena: aritmetička sredina standardna devijacija (SD), minimalna (min), maksimalna (max) vrijednost, koeficijent varijabilnosti (V\%), standardna greška aritmetičke sredine (SE). Razlike su testirane ANOVA i post-hoc Newman-Keuls testom, aritmetičke sredine koje dijele isto slovo su statistički značajne na nivou od 5\%, vrijednosti koje imaju (-) nisu značajne

\begin{tabular}{|l|c|c|c|c|c|c|}
\hline \multirow{2}{*}{ Parameters } & \multicolumn{3}{|c|}{ Formica rufa $\mathrm{N}=122$} & \multicolumn{3}{c|}{ Formica polyctena $\mathrm{N}=3$} \\
\cline { 2 - 7 } & $\begin{array}{c}\text { average } \pm \text { SD } \\
(\min , \max )\end{array}$ & $\mathrm{V} \%$ & $\mathrm{SE}$ & $\begin{array}{c}\text { average } \pm \text { SD } \\
(\min , \mathrm{max})\end{array}$ & $\mathrm{V} \%$ & $\mathrm{SE}$ \\
\hline Altitude & $\begin{array}{c}1291.2^{\mathrm{a}} \pm 82.9 \\
(981.0,1611.0)\end{array}$ & 6.4 & 7.5 & $\begin{array}{c}972.4^{\mathrm{a}} \pm 1.5 \\
(971.0,974.0)\end{array}$ & 0.16 & 0.88 \\
\hline $\mathrm{R} 1$ & $\begin{array}{c}141.3 \pm 60.4 \\
(25.0,340.0)\end{array}$ & 42.8 & 5.5 & $\begin{array}{c}83 \pm 56.9 \\
(20,130)\end{array}$ & 68.2 & 32.8 \\
\hline $\mathrm{R} 2$ & $\begin{array}{c}120.2 \pm 60.8 \\
(10.0,280.0)\end{array}$ & 50.6 & 5.5 & $\begin{array}{c}82 \pm 59.7 \\
(15,130)\end{array}$ & 73.0 & 34.4 \\
\hline $\mathrm{K} 1$ & $\begin{array}{c}99.5 \pm 56.6 \\
(10.0,220.0)\end{array}$ & 56.9 & 5.1 & $\begin{array}{c}72 \pm 53.0 \\
(15,120)\end{array}$ & 73.9 & 30.6 \\
\hline $\mathrm{K} 2$ & $\begin{array}{c}57.5 \pm 30.0 \\
(10.0,160.0)\end{array}$ & 52.2 & 2.7 & $\begin{array}{c}72 \pm 56.2 \\
(10,120)\end{array}$ & 78.4 & 32.4 \\
\hline
\end{tabular}

In order to examine association of wood ant to conifer trees we analyzed number of colonies that were built up a tree. In our research we detected that $56 \%$ of Formica rufa colonies and $70 \%$ of F. rufa phenotype were not leaning up the trees. Colonies of Formica rufa were most commonly built on south, south-east expositions $82 \%$. All colonies of Formica polyctena were built on plane terrain.
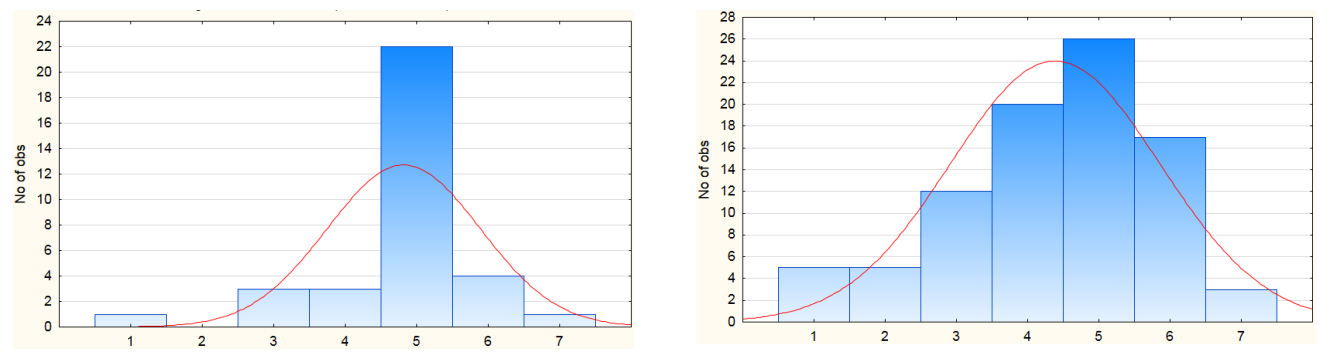

Graph 5-6: Distribution of Formica rufa (left) and F. rufa phenotype (right) number of colonies in regard to density of trees in habitat

Grafikon 5-6: Distribucija nalaza kolonija vrste Formica rufa (lijevo) i F. rufa fenotipa (desno) u odnosu na gustinu stabala u staništu 
Multivariate PCA analysis of ecological parameters indicates that first two principal components describe $52 \%$ of variability between Formica rufa, F. rufa phenotype and $F$. polyctena. In construction of first principal component mostly participate variable: habitat type 0.61 , altitude -0.51 , exposition -0.50 . Second principal component is constructed mostly by: habitat density -0.75 , construction of colonies up a tree 0.55 , position of colonies in regard to road 0.31 .

In investigated area wood ants show significant association with dens wood complexes. In closed woodlands number of colonies is higher and polydomy was observed in Formica rufa and F. polyctena. Colonies of Formica rufa on forest edges and fragmented woodland habitats were single. In deep and not fragmented wood complexes colonies were polydome and with large density. In Lavljak area, (Mt. Ozren), with fragmented woodland habitats, number of Formica rufa colonies in transect was 8/1000 m. Highest density of Formica rufa colonies was found on Mt. Perun 30/1000 m. Positive linear correlation between density and number of colonies was detected, but this correlation was not statistically significant at 5\% level. Maximal number of Formica rufa colonies decline after its maximum was reached in woodland habitats with 25-30 trees in 100 meter transect. There are two possible explanations for colony number decline in woodlands with higher tree density. In closed woodlands lower temperature is correlated with low insolation level. Second reason was relatively small number of sites with dens woodlands since Formica rufa colonies were dominantly built near wood roads $(70 \%)$. In our research we found that clearings in forest and wood roads were more attractive for wood ants probably due higher insolation.

In our investigation we detected that woodland roads and clearings in forest have positive effect on distribution and density of Formica rufa colonies. Formica rufa colonies were detected by roads that go through dense forests.

\section{CONCLUSIONS - Zaključci}

Central Bosnia is inhabited by five species belonging to subgenus Formica. In narrow sense Formica rufa group in Bosnia and Herzegovina include two species Formica rufa Linnaeus, 1761 and $F$. polyctena Förster, 1850.

Analysis of seta number in Formica rufa indicates that all samples have low hairiness in compare to central and north European populations. Bosnian wood antsô populations have below average hair number on occiput and pronotum when compared to central and north European populations.

Morphometric analysis of body indicates that Formica rufa less hairy phenotype has much more similarity to Formica rufa than to $F$. polyctena. 
Morphological investigations of reproductive caste should also be conducted in order to solve question of interbreeding between Formica rufa and F. polyctena in Bosnia and Herzegovina.

Further investigations should be conducted in wider area of Dinaric Alps in Bosnia and Herzegovina in order to sample and confirm presence of clear morphological phenotypes of Formica rufa.

\section{REFERENCES - Literatura}

Agostk. D., Collingwood, C. A. (1987): A provisional list of the Balkan ants (Hym. Formicidae) with a key to the worker caste. II. Key to the worker caste, including the European species without the Iberian.

Agostk. D., Collingwood, C. A. (1987): A provisional list of the Balkan ants (Hym. Formicidae) and a key to the worker caste. I. Synonymic list.Mitteilungen der Schweizerischen Entomologischen Gesellschaft, 60, 51-62

Agostk, D., Schultz, T., Majer, J. D. (Eds.). (2000). Ants. Smithsonian Instiution Press.

BrALKKo, G. (2006): Review of the ant fauna (Hymenoptera: Formicidae) of Croatia. Acta entomologica slovenica, 14, 2.

Bratko, G. (2007): Checklist of the ants of Slovenia (Hymenoptera: Formicidae). Natura Sloveniae, 9(1), 15-24.

Collisgwood, C. A. (1979): The Formicidae (Hymenoptera) of Fennoscandia and Denmark. Scandinavian Science Press.

Collikgawood, C. A., Agostķ D. (1986): Taxonomy and zoogeography of the Formica rufa species group. In Supplement to the Workshop ñTaxonomy and Zoogeography of the Formica rufa Species Groupò Held at the 10th International Congress of IUSSI, Munich.

CZECHOWSKk W. (1996): Morphometric Characteristics of Formica polyctena Foerst. and Formica rufa L.(Hymenoptera, Formicidae) from the Gorce Mts. InAnnales Zoologici (Vol. 46, pp. 125-141).

Gösswald, K. (1981): Artunterschiede der Waldameisen in Aussehen, Lebensweise, Organisation, Verhalten, Nest- und StraOenbau, Okologie und Verbreitung. Merkblatter zur Waldhygiene, 1:1-32.

Karaman, M. (2004): Checklist of known species of ants (Hymenoptera, Formicidae) in the fauna of Montenegro. Natura Montenegrina, 3, 83-92.

LUTERĠEK, D. (1970). Mravlja fauna Igmana sa narol itim osvrtom na privredno vaǵne vrste. Radovi gimarskog fakulteta i instituta za ġmarstvo u Sarajevu, 12(4 ï 6). 
Petrov, I. (2006). Ants of Serbia. Serbian Academi of Sciences and Arts. Department of Chemical and Biological Sciences 4, 1-133.

Radchenko, A. (2015). Fauna Europaea: Hymenoptera, Formicidae. Fauna Europaea version 2.6.

SElfERT, B. (1991): The phenotypes of the Formica rufa complex in East germany. Abhandlungen und Berichte des Naturkundemuseums Görlitz, 65, 1-27.

Sekfert, B. (2007). Die Ameisen Mittel-und Nordeuropas (pp. 204-320). Tauer: Lutra Verlags-und Vertriebsgesellschaft.

SEkEERT, B. (1992): Formica nigricans Emery, 1909 ï an ecomorph of Formica pratensis Retzius, 1783 (Hymenoptera, Formicidae). ठ Entomol. Fennica 2: 217ї 226.

THOR, G., (1998): Red-listed lichens in Sweden: habitats, threats, protection, and indicator value in boreal coniferous forests. Biodiversity and Conservation, 7(1), $59-72$.

VESNḰ, A. (2011): Revised systematic list of ants of Bosnia and Herzegovina. In: Lelo, S. (ed.): Fauna of Bosnia and Herzegovina ï A biosystematic review. 7th amended and updated internal edition of Society for Inventarisation and protection of animals, Ilijag் Canton Sarajevo, pp: 205-207.

\section{SAŽETAK}

Ġumski mravi iz podroda Formica imaju vaǵnu ulogu u ekologiji l etinarskih ġıma. Poseban znal aj imaju vrste iz grupe Formica rufa. Kolonije imaju veliku biomasu, sa promjerom vel im od dva metra. Vrste Formica rufa i Formica polyctena su ekoloǵki vezane za ġme smrl e i jele. Bioloǵki podaci o vrstama Formica rufa i $F$. polyctena u Bosni i Hercegovini su na nivou faunistil kih podataka.

U radu su prezentirani podaci o diverzitetu ġmskih mrava u Bosni i Hercegovini. Prvi nalaz vrste Formica truncorum Fabricius, 1804 sa podrul ja Duboǵice prijavljen je za Bosnu i Hercegovinu. Ukupan broj vrsta iz podroda Formica u fauni podignut je na pet. Istraǵivanjem lokaliteta u centralnoj Bosni utvrĹeno je prisustvo vrste Formica rufa Linnaeus, 1761 na planinama Trebevil, Ozren i Perun. Formica polyctena sakupljena je samo na planini Lemerska.

Rad ima za cilj analizu diverziteta podroda Formica i populacione strukture vrsta Formica rufa i $F$. polyctena. Na osnovu morfometrijskih pokazatelja izvrǵena je diferencijacija blisko srodnih vrsta Formica rufa i $F$. polyctena. Populacija vrste Formica rufa podijeljena je prema stepenu dlakavosti na dva fenotipa. Uzorci identificirani kao tipski fenotip Formica rufa prema stepenu dlakavosti su na donjoj granici raspona dlakavosti specifi! nog za vstu. Na osnovu intenziteta dlakavosti u populaciji Formica rufa se izdvajaju intermedijerni fenotipovi. Uzorci Formica rufa 
sa podprosjel nim brojem dlaka imaju osobine opisanog hibrida Formica rufa $x$ polyctena ili dlakavog fenotipa Formica polyctena.

Diskriminantnom funkcijom uzorci manje dlakavog fenotipa Formica rufa nisu izdvojeni u poseban klaster. Na osnovu simpatril nog prostornog rasporeda pronaL'enih fenotipova bez geografske agregacije najvjerovatnije je rijel o ekomorfima. $\mathrm{Na}$ istrağivanom podrul ju centralne Bosne nizak stepen urbanizacije i minimalni turistil ki kapaciteti nisu prepoznati kao osnovini faktori rizika za gimske mrave.

1 - Mr. sc. Adi Vesniĺ, Faculty of Natural Sciences and Mathematics, Biology Department, 71000 Sarajevo, Bosnia and Herzegovina, vesnic.adi@gmail.com

2 - Prof. dr. Rifat Ǵrrijelj, Faculty of Natural Sciences and Mathematics, Biology Department, 71000 Sarajevo, Bosnia and Herzegovina

3 - Prof. dr. Sadbera Troǵí -Borovac, Faculty of Natural Sciences and Mathematics, Biology Department, 71000

Sarajevo, Bosnia and Herzegovina

* Acknowledgements

The study was financially supported by the Rufford Small Grant. 